\title{
The Histopathological Spectrum and Clinico-Pathological Concordance in 85 Cases of Skin Biopsy: A Single Center Experience
}

\author{
Jha HK ${ }^{1}$, Pokharel $A^{2}$ \\ ${ }^{1}$ College of Medical Science, Bharatpur, Chitwan, Nepal; ${ }^{2}$ Consultant Dermatologist, Bharatpur, Chitawan, Nepal
}

\begin{abstract}
Introduction: Skin disease is common in developing countries but the spectrum varies widely. Skin biopsy is an important tool in confirming or establishing diagnoses in cases with diagnostic dilemmas. And, there may be a discrepancy between clinical and pathological diagnoses.

Objectives: To determine the spectrum of histological diagnoses in skin disease and to know the consistency between clinical and pathological diagnoses.

Materials and methods: A retrospective review of histological and clinical diagnoses was done at a multi-specialty hospital. A total of 85 cases were included in the study. Clinical and histological data along with demographic details, the site of the biopsy was noted. Concordance between clinical and pathological diagnosis was determined as concordant, partially concordant and discordant.

Results: There were $60 \%$ female and $40 \%$ male patients out of 85 biopsies. The most common age group was $31-40$ years and the mean age was 41.24 years. The most common histological spectrum was infectious group in $29.4 \%$, followed by papulosquamous disorders in $22.4 \%$ and eczema in $12.9 \%$ cases. The concordance between clinical and pathological diagnosis was $60 \%$, while partial concordance was seen in $16.5 \%$ and discordance was seen in $23.5 \%$ cases.

Conclusion: Infections followed by papulosquamous disease and eczema were the three most common groups of histological diagnosis. This study also found an overall concordance of $76.5 \%$ and discordance in $23.5 \%$ between clinical and histopathological diagnosis.
\end{abstract}

Key words: Biopsy; Retrospective study; Skin disease

\section{Introduction}

kin diseases are diverse however, most of the conditions are diagnosed based on visual examination and clinical history. Skin biopsy is an important tool to confirm the clinical diagnosis in cases of diagnostic dilemmas. Since it is performed only in specific cases, a high diagnostic yield is always desired. This depends on number of factors like biopsy technique, site of biopsy, adequate sample, appropriate clinical and physical findings etc. Sometimes the pathological findings are quite different than the clinical diagnosis which leads to clinico-pathological discordance. There are very few studies on clinico-pathological concordance and none ever reported from Nepal. The aim was to study the

Funding: No

Conflict of Interest: No

Address of Correspondence

Dr. Harendra Kumar Jha

College of Medical Science, Bharatpur, Chitwan, Nepal

E-mail: harendrajha@gmail.com histological spectrum of skin biopsies and also assess the concordance between clinical and histological diagnoses.

\section{Materials and methods}

This was a retrospective, cross-sectional study conducted in multi-specialty hospital, over a period of

Submitted: $18^{\text {th }}$ December 2020

Accepted: $6^{\text {th }}$ February 2021

Published: $20^{\text {th }}$ February 2021

How to cite this article

Jha HK, Pokharel A. The Histopathological Spectrum and Clinico-Pathological Concordance in 85 Cases of Skin Biopsy: A Single Center Experience. Nepal Journal of Dermatology, Venereology \& Leprology 2021;19(1):21-25. https://doi. org/10.3126/njdvl.v19i1.33534.

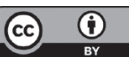

Licensed under CC BY 4.0 International License which permits use, distribution and reproduction in any medium, provided the original work is properly cited. 
2 years (June 2013 to May 2015). The analysis of 85 skin biopsy was done based on the available records from the record section. All biopsy involved punch biopsy technique. All samples were processed routinely with Hematoxylin and eosin ( $\mathrm{H}$ and $\mathrm{E}$ ) stain. Special stains like Ziehl-Neelsen (ZN) and periodic acid-Schiff (PAS) stains were used whenever appropriate. The demographic details like age and sex were noted. Other relevant information like the site of biopsy, provisional and differential diagnosis, histopathological diagnosis, any special stain beside $\mathrm{H}$ and $\mathrm{E}$ stain was also recorded. Biopsy with incomplete information and unconfirmed diagnosis was not included. All the biopsy was read and reported by a dermatopathologist. Concordance was categorized under 3 categories namely

i) Concordant - when both provisional and histological diagnosis exactly matched

ii) partially concordant - when histological diagnosis matched with one of differential diagnosis

iii) discordant - when clinical and histological diagnosis differed completely

The histological spectrum of diagnoses was categorized into 7 categories namely, infections, eczema/ dermatitis, papulosquamous disease, connective tissue disorder, benign tumors, pigmentary disorder and miscellaneous group. The data were entered in Microsoft Excel and statistical analysis was done with SPSS version 22 along with Fisher's exact test. Study was approved by the ethical committee.

\section{Results}

Out of 85 biopsies, $60 \%$ were from female patients while it was $40 \%$ from male patients. The age of the patients ranged from 7 to 84 years and the mean age was 41.24 years. Age and sex details are given in table 1 . The site of biopsy in descending order was lower limb (36.5\%), upper limb (25.9\%), trunk (24.7\%) and face (12.9\%). Most common histological spectrum was infectious group $(25,29.4 \%)$, followed by papulosquamous disorder $(19,22.4 \%)$ and eczema/ dermatitis $(11,12.9 \%)$. Among infectious diseases, the most common diagnosis was Hansen's disease (12, $14.1 \%)$, followed by lupus vulgaris $(7,8.23 \%)$. Psoriasis was most common among papulosquamous disorder $(8,9.41 \%)$ followed by lichen planus $(5,5.88 \%)$. Other histological spectrum included benign tumor $(6,7.05 \%)$, connective tissue disorder (3,3.52\%), pigmentary disorder (2, 2.35\%). The miscellaneous group included the rest of the diagnosis $(19,22.35 \%)$. The spectrum of histological diagnoses and their sex-wise distribution is given in table 2. Concordance between clinical and histological was seen as concordant in $60 \%$ cases, partial concordant in $16.5 \%$ cases and discordant in $23.5 \%$ cases. (Figure 1). Thus, the overall concordance was seen in $76.5 \%$ cases. Concordance rate was significantly high only in Hansen's disease. (Table 3)

Table 1: Age and sex distribution of patients

\begin{tabular}{|c|c|c|c|}
\hline Age group (years) & Males & Females & Total \\
\hline $0-10$ & 1 & 0 & 8 \\
\hline $11-20$ & 4 & 4 & 17 \\
\hline $21-30$ & 5 & 12 & 21 \\
\hline $31-40$ & 7 & 14 & 10 \\
\hline $41-50$ & 3 & 7 & 17 \\
\hline $51-60$ & 9 & 8 & 11 \\
\hline$>60$ & 5 & 6 & 85 \\
\hline
\end{tabular}

Table 2: Table showing individual disease group and concordance

\begin{tabular}{|l|c|c|c|}
\hline Group & Concordance & Partial concordance & Discordant \\
\hline Infections & 20 & 2 & 3 \\
\hline Eczema & 6 & 2 & 1 \\
\hline Papulo-squamous disease & 13 & 5 & 0 \\
\hline Connective tissue disease & 1 & 2 & 1 \\
\hline Pigmentary disease & 1 & 0 & 4 \\
\hline Soft tissue tumor & 2 & 0 & 8 \\
\hline Miscellaneous & 8 & 3 & $\mathbf{2 0}$ \\
\hline Total & $\mathbf{5 1}$ & $\mathbf{1 4}$ & 3 \\
\hline
\end{tabular}


Table 3: Concordance in top 5 histopathological diagnosis

\begin{tabular}{|l|c|c|c|c|}
\hline Diagnosis & Concordant & Partially concordant & Discordant & P-value (Fisher's exact test) \\
\hline Hansen's disease & 12 & 0 & 0 & 0.008 \\
\hline Psoriasis & 6 & 2 & 0 & 0.246 \\
\hline Lupus vulgaris & 7 & 0 & 0 & 0.127 \\
\hline Lichen planus & 4 & 1 & 0 & 0.549 \\
\hline Granuloma annulare & 3 & 0 & 1 & 1.00 \\
\hline
\end{tabular}

60

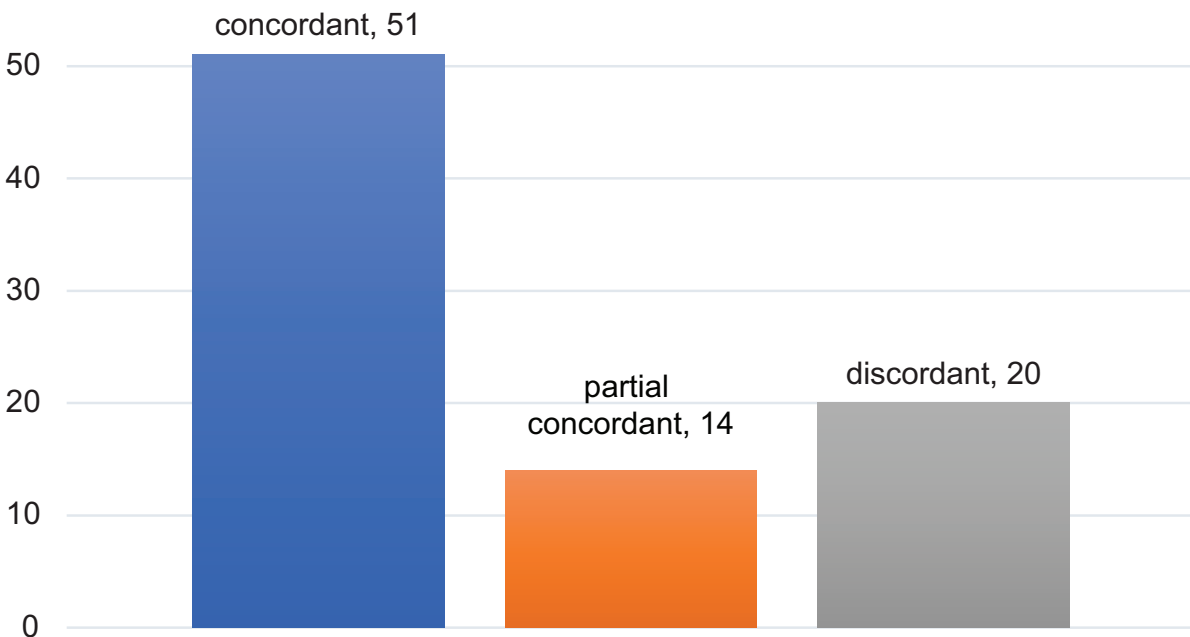

Figure 1: Bar diagram showing concordance between clinical and pathological diagnosis

\section{Discussion}

Skin biopsy is one the most important diagnostic tool performed by a dermatologist in cases of diagnostic dilemma. However, the interpretation of histopathological finding remains a challenge even for the most experienced pathologist. Adequate clinical information, clinical photograph all help to reach to a definitive diagnosis. There are not many studies reported from Nepal on the histological spectrum of skin disease and none about the concordance of clinical and pathological diagnosis.

This study found that more cases of biopsy were performed in females (60\%) than males (40\%). This was similar to the finding reported by Al-saif et al who found more number of biopsy in females (58.1\%) than males. ${ }^{1}$ Also, a study from Nepal had found female predominance (1.08:1). ${ }^{[2]}$ The most common age group in which biopsy was performed was 31-40 years with a mean age of 41.24 years. It was similar to the finding reported by Adhikari et al. ${ }^{2}$ While Chalise et al reported $41-50$ years as the most common age group. ${ }^{3}$ The most common histopathological spectrum was infectious disorders (29.4\%). Gupta et al also reported infectious disease as the most common spectrum (34.58\%). ${ }^{4}$ While two other studies reported papulosquamous disease as the most common histopathological spectrum. ${ }^{1,5}$ Vesicobullous and vesicopustular disease were most commonly seen in the study reported from Nepal. ${ }^{3}$ These findings reflect the wide histopathological spectrum that is seen in a particular country and region.

Among infectious diseases, Hansen's disease was the most common infection $(12,14.1 \%)$, followed by lupus vulgaris $(7,8.23 \%)$. Other studies also have found Hansen's disease as the most common bacterial infection. ${ }^{2,4,6}$ A Nigerian study had reported viral infection as the most common infectious etiology. ${ }^{7}$ In Nepal and India, Hansen's disease is still found commonly as shown by our study. The use of histopathology is to confirm and identify the spectrum of leprosy, thus helping the clinician to decide the choice of multi-drug therapy (MDT). Slit-skin smear still remains a valuable tool in multibacillary disease. Lupus vulgaris is the next common mycobacterial skin infection. A similar observation is made in other studies. ${ }^{2,6}$ Few cases of dermatophyte infection, leishmaniasis and chromoblastomycosis were also 
diagnosed in our study. Papulosquamous disease was the second most common group of histopathological diagnoses (22.35\%). Psoriasis was the most common among papulosquamous diseases followed by lichen planus and pityriasis rosea. Similar observations were made by Agrawal et al ${ }^{8}$ and Reddy et al. ${ }^{9}$ However, Ogun et al found lichenoid dermatitis as the most common disorder among papulosquamous disease, ${ }^{7}$ while a study from Nepal, found Erythema dyschromicum perstans as the commonest one. ${ }^{2}$ Chronic eczema was the most common eczema reported followed by few cases of prurigo nodularis, pompholyx and seborrheic dermatitis. Adhikari et al reported spongiotic dermatitis as the most common form of eczema. ${ }^{2}$ Under pigmentary disorder, 2 cases of post-inflammatory hypopigmentation were seen, while 2 cases of morphea and 1 case of discoid lupus erythematosus were seen under connective tissue disorder. Connective tissue disorder frequency (3.52\%) was similar to Adhikari et al $(2.4 \%){ }^{2}$ Under the miscellaneous category, granuloma annulare (GA) was the most frequent (4.7\%). Our study did not find a single case of malignancy. It may be explained due to the fact that the study site is a general dermatology clinic and also the presence of a prominent cancer hospital nearby.

Our study found concordant clinico-pathological findings in $60 \%$ and partial concordant in $16.5 \%$ cases, hence there was an overall concordance in $76.5 \%$ cases. It was discordant in $23.5 \%$ of cases. Also, we found that the concordance was significantly high only in Hansen's disease. Sa et al found overall concordance in $86.5 \%$ and discordance in $13.5 \%$ cases. ${ }^{10}$ Aslan et al also reported concordance in $76.8 \%$ cases in discordance in $23.2 \%$ cases. $^{11}$ They also noted that providing adequate clinical descriptive information in pathology requisition form increases the probability of making an accurate diagnosis. Balasubramanian et al reported a concordance rate of $59.8 \%$, minor discordance in $7.6 \%$ and discordance in $30.9 \%$ cases. ${ }^{12}$ They observed that the concordance rate was high in eczematous and lichenoid disorder less in granulomatous and pigmentary disorder. Cerroni et al found that there was a $16.6 \%$ overall increase in accuracy of diagnosis when a clinical picture of the patients was provided to the dermatopathologists. ${ }^{13}$ Malik et al found 61.01\% clinicopathological concordance. ${ }^{14}$ They also concluded that the clinical photograph of the patient helps in better correlation at the time of diagnosis. Our study findings are also consistent with the findings of the above studies. In our personal experience, the concordance between clinical and histopathological diagnosis can be increased by providing adequate clinical information on requisition form and providing relevant clinical picture of the lesion to the dermatopathologist.

\section{Conclusion}

Skin biopsy is an invaluable tool in cases of diagnostic dilemmas. If performed adequately and accurately, it helps in reaching clinical diagnosis easily. This study has brought out the histopathological spectrum in a specialty hospital setup. Infections followed by papulosquamous disease and eczema were the three most common groups of histological diagnosis. Our study also found an overall concordance of $76.5 \%$ and discordance in $23.5 \%$ of cases. Hence, again emphasizing the important role of skin biopsy in establishing a diagnosis for proper management.

4. Gupta P, Karuna V, Grover K, Rathi M, Verma N. The histopathological spectrum of skin diseases with emphasis on clinicopathological correlation: A prospective study. IP J Diagn Pathol Oncol 2018;3(2):91-95. https://doi.org/10.18231/25813706.2018.0020

5. Azam F, Munir S, Ashraf A, Imran A, Rashid T. Histopathological analysis of skin biopsies in a tertiary care setting. J Pak Assoc Dermatol 2017;27(2):154-57.

6. George V, Sowmya S, Krishnan S. A histopathological study of skin biopsy specimens in a tertiary care hospital with a keynote on clinicopathological correlation. Annals Pathol Lab Med 2020;7(1): A39-45. https://doi. org/10.21276/apalm.2670 
7. Ogun G, Okoro O. The spectrum of non- neoplastic skin lesions in Ibadan, Nigeria: a histopathologic study. Pan Afr Med J 2016;23. https://doi. org/10.11604/pamj.2016.23.221.9372

8. Agrawal S, Mishra K, Gupta C. Histopathological spectrum of non-infectious erythematous, papulosquamous lesions: at a tertiary care institute. Int J Res Med Sci 2018;6(6):207275. https://dx.doi.org/10.18203/2320-6012. ijrms20182291

9. Reddy B, Nalini M. Histopathological spectrum of non-infectious erythematous, papulosquamous lesions. Asian Pac J Trop Med 2014;1(4S):28-34. https://dx.doi.org/10.21276/apjhs.2014.1.1s.6

10. Sa D, Kumar P. Clinicopathological consistency in diagnosis of skin disorders: a retrospective study of 371 histopathology reports. J Pak Assoc Dermatol 2016;26(2):96-8.

11. Aslan C, Gktay F, Mansur A, Aydngz k, Gne $\mathrm{P}$, Ekmeki T. Clinicopathological consistency in skin disorders: A retrospective study of 3949 pathological reports. J Am Acad Dermatol 2012;66(3):393-400. https://dx.doi. org/10.1016/j.jaad.2010.12.031

12. Balasubramanian $P$, Chandrashekar L, Thappa D, Jaisankar T, Malathi M, Ganesh R et al. A retrospective audit of skin biopsies done in a tertiary care center in India. Int J Dermatol 2015;54(8):939-943. https://dx.doi.org/10.1111/ ijd.12718

13. Cerroni L, Argenyi Z, Cerio R, Facchetti F, Kittler $\mathrm{H}$, Kutzner $\mathrm{H}$, et al. Influence of evaluation of clinical pictures on the histopathologic diagnosis of inflammatory skin disorders. J Am Acad Dermatol 2010;63(4):647-52. https://dx.doi. org/10.1016/j.jaad.2009.09.009

14. Malik A, Siraj F, Shruti S, Gupta P, Khullar G, Ramesh V. Clinicopathological concordance in 2216 cases of skin biopsy over one year: An Indian experience. Cureus 2020;12(4):e7752. https://dx.doi.org/10.7759/cureus.7752 